\title{
Über Bleitetrachlorid
}

von

\section{H. Friedrich.}

Aus dem chemischen Institute der k. k. Universität in Graz.

(Vorgelegt in der Sitzung am 13, Juli 1893.)

Das Blei wird in unorganischen Verbindungen in der Regel als zweiwerthiges Element betrachtet und es hat auch zweifellos Ähnlichkeit mit Elementen von ausgesprochener Bivalenz, wie den alkalischen Erden. Das Hyperoxyd lässt wohl vermuthen, dass das Blei auch als vierwerthiges Element auftreten könne, ist aber hiefür nicht beweisend.

Dafür wurde die Tetravalenz des Bleies durch die Entdeckung seiner Alkylverbindungen ausser Zweifel gesetzt.

A. Butlerow ${ }^{1}$ bestimmte bekanntlich schon 1863 die Dampfdichte des von Cahours entdeckten Bleitetramethyls und fand, dass sie der Formel $\mathrm{Pb}\left(\mathrm{CH}_{3}\right)_{4}$ entspreche, wodurch die Vierwerthigkeit des Bleies zur Thatsache wurde.

Weiterhin hat sich die Zahl analog zusammengesetzter metallorganischer Verbindungen vermehrt, wir kennen z. B. das Bleitrimethylchlorid $\mathrm{Pb}\left(\mathrm{CH}_{3}\right)_{3} \mathrm{Cl}$, das Bromid und Jodid, Bleiteträthyl $\mathrm{Pb}\left(\mathrm{C}_{2} \mathrm{H}_{5}\right)_{4}$, Bleitriäthyljodür $\mathrm{Pb}\left(\mathrm{C}_{2} \mathrm{H}_{5}\right)_{3} \mathrm{~J}$, Bleitetraphenyl $\mathrm{Pb}\left(\mathrm{C}_{6} \mathrm{H}_{5}\right)_{4}$, Bleidiphenylchlorid $\left(\mathrm{C}_{6} \mathrm{H}_{5}\right)_{2} \mathrm{PbCl}_{2}$, Bleidiphenyloxyd $\left(\mathrm{C}_{6} \mathrm{H}_{5}\right)_{2} \mathrm{PbO}$ und seine Derivate, und endlich das $p$ - Bleitetratolyl $\left(\mathrm{Pb}\left(\mathrm{C}_{6} \mathrm{H}_{4} . \mathrm{CH}_{3}\right)_{4}, \quad p\right.$-Bleiditolylchlorid $\mathrm{Cl}_{2} \mathrm{~Pb}\left(\mathrm{C}_{6} \mathrm{H}_{4} \cdot \mathrm{CH}_{3}\right)_{2}$ etc. etc.

So war man in der organischen Chemie bereits im Besitze einer ganzen Reihe von Verbindungen, in denen das Blei als vierwerthiges Element auftritt, während die anorganische keine

1 Jahresber. für Chemie, 1863, 476. 
aufzuweisen hatte. Erst in den letzten Jahren macht sich auch hier ein Fortschritt bemerkbar.

Im Jahre 1885 gelang es Nikoljukin, das Superchlorid aus der Lösung von $\mathrm{PbO}_{2}$ in kalter Salzsäure in eine wägbare Form zu bringen, indem er es an Ammoniumchlorid band und ein analysirbares, gut charakterisirtes Doppelsalz erhielt. Nikoljukin bestimmt in dieser Verbindung nur das Verhältniss von Chlor zu Blei und sucht daraus den Beweis für die Existenz des Bleitetrachlorids zu liefern, die schon von anderen Chemikern vermuthet worden ist. ${ }^{1}$

Die genaue Zusammensetzung der Doppelverbindung hat er jedoch nicht ermittelt.

Schon im Jahre 1889 habe ich in etwas anderer Art, nämlich durch Einwirkung von Chlor auf eine Lösung von Bleidichlorid in concentrirter Salzsäure, eine Flüssigkeit dargestellt, die nach ihren Reactionen das Bleitetrachlorid enthielt, und aus dieser durch Zusatz von Ammoniak oder Chlor-

1 In der Literatur finden sich folgende den Gegenstand betreffende Angaben:

E. Millon (1842). J. Pharm. et Chimie, T. I, p.299; Ant. Chem. Pharm., 44, 236 ; Compt. rend., XXVIII, 42; Jahresber. für Chem., 1849, 254; Pharm. Centr., 1849, 208; Inst. 1849, 29.

Sobrero-Selmi (1850). Ann. chim. phys., XXIX, [3], 161; Jahresber. für Chem., 1850, 322 (III); Centralbl., 1850, 615-616; Ann. Chem. Pharm., LXXVI, 234.

Rivot, Beudant, Daguin (1853), Ann. min. [5], IV, 239; Jahresber. für Chem., 1853, VI, 370.

J. Niklés (1866). Compt. rend., LXIII, 1118; Jahresber. für Chem., 1866, 232 ; Ann. chim. phys., [4], X, 323; Inst., 1866, 412; J. Pharm., [4], V.91, J. pr. Chem., C, 494; Zeitschr. Chem., 1867, 45; Sill. Ann. J., [2], XLIII, 94.

H. Schiff (1875), Berl. Berichte, 1875, 1198.

W. W. Fisher (1879), Berl. Berichte, 1879, (XII), 849; Chem. Soc. J., 35, 282; Jahresber. Chem., 1879, 279.

O. Seid1 (1879). Jahresber. Chem., 1879, 279; J. pr. Chem., 128, [20], 205.

A. Ditte (1880). Compt. rend., 91, 765; Jahresber. für Chem., 1880, 328 ; Berl. Berichte, 1880, 2411.

J. Nikoljukin (1885). J. der russ. phys.-chem. Gesellsch., 1885, (1), 200-207; Berl. Berichte, 1885, R., 370.

H. Friedrich (1889). Sitzungsber. der königl. böhm. Gesellschaft der Wissenschaften, $1889,161$.

A. Classen und B. Zahorski (1893). Z. für anorg. Chem., IV, Heft 1 und $2,100$. 
ammonium ein krystallinisches Doppelsalz abgeschieden, dessen Analyse gut auf die Formel $\mathrm{PbCl}_{4} \cdot 2 \mathrm{NH}_{4} \mathrm{Cl}$ stimmte. Wie ich in einer vorläufigen Mittheilung damals kurz angegeben habe, ist es mir auch gelungen, das Bleitetrachlorid zu isoliren.

Vor Kurzem ist eine Untersuchung der Herren A.Class en und B. Zahorski erschienen, in.welcher sie das von Niko1 jukin (1885) und mir (1889) entdeckte Doppelsalz beschreiben und analysiren. Die Analysenresultate und demgemäss die Formel der Herren Classen und Zahorski weichen von den meinen wesentlich ab und darum glaube ich auch, den Theil meiner Arbeit, der sich auf das Doppelsalz bezieht, im Wesentlichen beschreiben zu müssen.

\section{Darstellung von Bleitetrachloridchlorammonium.}

Aus reinem Bleicarbonat bereitetes Bleidichlorid wurde im Verhältniss von etwa $1: 20$ in starke Salzsäure eingetragen und in einem kühlen Raume $\left(10-15^{\circ} \mathrm{C}\right.$.) mit Chlor gesättigt. Allmälig erfolgt fast vollständige Lösung und man erhält eine dunkelgelbe, stark nach Chlor riechende klare Flüssigkeit. Ein geringer Bodensatz von unverändertem Bleichlorid kann durch Zufügen von concentrirter Salzsäure und neuerliches Einleiten von $\mathrm{Cl}$ vollständig in Lösung gebracht werden.

$\mathrm{Zu}$ der vollkommen klaren Flüssigkeit fügt man die für zwei Moleküle berechnete Menge von Chlorammonium, die in der zehnfachen Menge Wassers gelöst ist, hinzu. Beide Flüssigkeiten werden vor dem Zusammengiessen in Eis gekühlt. Nach kurzer Zeit scheidet sich ein krystallinischer Niederschlag von gelber Farbe (mit einem leichten Stich ins Grüne) ab. Nach etwa einer Viertelstunde wird die überstehende Flüssigkeit abgezogen und die Krystallisation auf einem gleichfalls mit Eis gekühlten Filtrirtrichter mit der Saugpumpe von der Mutterlauge möglichst befreit. Das Doppelsalz.wird dann auf dem Filter mit in Eis gekühltem Alkohol, welcher in der Kälte nicht einwirkt und auch sehr wenig löst, einigemale abgespült, hierauf zwischen Filterpapier bei gewöhnlicher Temperatur getrocknet. Bei dieser Manipulation ist die Ausbeute eine ziemlich bedeutende. Ich erhielt im Durchschnitt auf $30 \mathrm{~g}$ verwendetes $\mathrm{PbCl}_{2}$ $31 \mathrm{~g}$ des Doppelsalzes gegen $49 \mathrm{~g}$ der theoretischen Berechnung. 
Nach den von mir gesammelten Erfahrungen ist es nicht räthlich, zur Ausfällung concentrirte $\mathrm{NH}_{4} \mathrm{Cl}$-Lösungen anzuwenden. Mit solchen habe ich ein unreines Doppelsalz erhalten, bei dessen mikroskopischer Untersuchung Krystalle von $\mathrm{NH}_{4} \mathrm{Cl}$ nachzuweisen waren. Ich komme darauf noch zurück.

Von einer 10\% igen Ammoniumchloridlösung schadet dafür auch ein Überschuss nicht.

Das auf diese Weise gewonnene Doppelsalz zeigt im Allgemeinen die von Nikoljukin und neuerdings von ClassenZahorski beschriebenen Eigenschaften.

Der gelbe Körper ist nicht hygroskopisch, hat einen süsslich adstringirenden Geschmack und zeigt ausgeprägten krystallinischen Charakter. Er besteht aus kleinen Krystallen, die, unter dem Mikroskope betrachtet, deutlich Oktaëderflächen zeigen, auch Combinationen mit dem Würfel schienen vorzukommen. Es gelang mir, aus den Mutterlaugen von der Bereitung des Salzes, die sich in wohlverschlossenen Flaschen lange halten, grössere Krystalle $\mathrm{zu}$ bekommen. Die Laugen wurden in grossen Glasflaschen durch etwa zwei Monate starken Frösten ausgesetzt, wodurch sich die darin gelösten Salze in Krystallkrusten am Boden der Gefässe absetzen. Ich erhielt auf diese Weise Krystalle des Doppelsalzes von der Grösse eines Stecknadelkopfes, die sehr leicht von den anderen mitauskrystallisirten Salzen - wahrscheinlich $\mathrm{PbCl}_{2}$ und $\mathrm{NH}_{4} \mathrm{Cl}$ - getrennt werden konnten.

Herr Dr. Koechlin im Wiener Hofmuseum hatte die Freundlichkeit, mir über eine Probe dieser Krystallisation mitzutheilen:

Krystallsystem: tesseral.

Combination: Hexaëder mit Oktaëder, meist in ebenmässiger Ausbildung.

Die Flächen sind meist eben, aber so matt, dass sie im besten Falle sehr schwache, gewöhnlich aber gar keine wahrnehmbaren Reflexe geben.

Die Substanz ist einfach brechend."

Ich fühle mich verpflichtet, Herrn Dr. Koechlin hiefür meinen besten Dank auszusprechen. 
Das Salz enthält kein Krystallwasser und ist eine verhältnissmässig beständige Verbindung. Es zersetzt sich erst bei $120^{\circ}$ (Nikoljukin), wobei die gelbe Farbe in eine dunkelorangegelbe übergeht, dabei entweicht sichtlich Chlor und schliesslich sublimirt Ammoniumchlorid. Bleidichlorid bleibt bei vorsichtigem Erhitzen als pulverförmige, lockere Masse zurück und schmilzt bei steigender Temperatur zu einer gelben Flüssigkeit, welche nach dem Abkühlen zu Hornblei erstart.

Ich bestimmte wiederholt und mit verschiedenem Material den Gewichtsverlust bei dieser Zersetzung, d. i. ich wog das zurückgebliebene, noch nicht eingeschmolzene Bleidichlorid.

$\begin{array}{cccc}\text { Verlust an Gewicht } & & \text { Zurückbleibendes } \\ \text { Substanz } & \text { in Gramm } & \text { In Procenten } & \mathrm{Pb} \mathrm{Cl}_{2} \text { in Procenten } \\ \text { I.... } 0.44875 \mathrm{~g} & 0.17525 & 39.05 & 60.94 \\ \text { II....0.35075 g } & 0.1370 & 39.05 & 60.94 \\ \text { III....0.9774g } & 0.3810 & 38.98 & 61.01 \\ \text { IV.... } 0.5639 \mathrm{~g} & 0.2197 & 38.96 & 61.03\end{array}$

Diese Zahlen entsprechen einer Verbindung von der Formel $\mathrm{PbCl}_{4} \cdot 2 \mathrm{NH}_{4} \mathrm{Cl}$.

Die weiteren analytischen Bestimmungen geschahen folgendermassen:

Das Blei wurde als Bleisulfat gewogen. Die lufttrockene Substanz blieb mit wässeriger Schwefelsäure in der Kälte stehen, wobei die Zersetzung sehr langsam erfolgt, dann wurde abgedampft und wiederholt mit concentrirter Schwefelsäure abgeraucht.

$0.28175 \mathrm{~g}$ Substanz gaben $0.18725 \mathrm{~g} \mathrm{~Pb} \mathrm{SO}_{4}=45 \cdot 38 \% \mathrm{~Pb}$.

Die totale Chlormenge wurde auf zweifache Art bestimmt.

Bei der ersten Analyse wurde das Doppelsalz mit viel Wasser zersetzt. Nach schwachem Erwärmen setzte sich das $\mathrm{PbO}_{2}$ gut $\mathrm{ab}$ und wurde abfiltrirt, im Filtrat dann Chlor durch Titration bestimmt.

$0.1673 \mathrm{~g}$ Substanz enthielten $0.077647 \mathrm{~g} \mathrm{Cl}=46.41 \%$.

Bei der zweiten Bestimmung wurde das Doppelsalz durch eine Natriumcarbonatlösung unter gelindem Erwärmen zersetzt. Das Salz wandelt sich in einen hellbraunen Niederschlag um, 
der sich unter Abscheidung von $\mathrm{PbO}_{2}$ und $\mathrm{CO}_{2}$ zersetzt. Im Filtrat wurde das Chlor wie oben bestimmt.

$0.1595 \mathrm{~g}$ Substanz gaben $0.074286 \mathrm{~g} \mathrm{Cl}=46.57 \%$.

Das Mittel aus beiden Werthen ist $46: 49 \%$.

Bei Bestimmung des sogenannten activen Chlors wurden gleichfalls zwei Methoden in Anwendung gebracht.

Einmal wurde das Doppelsalz direct in eine Jodkaliumlösung eingetragen. Es erfolgt Zersetzung unter Abscheidung von Jod und Bildung von Bleijodid. Nachdem die Einwirkung augenscheinlich vollendet war, wurde auf $l l$ verdünnt und bis zur Klärung der Flüssigkeit stehen gelassen; hierauf wurden zweimal $250 \mathrm{~cm}^{3}$ dem Messkolben entnommen und darin die Jodmenge mittelst Natriumthiosulfatlösung bestimmt. $0.3992 \mathrm{~g}$ Substanz entsprechen $0.2221 \mathrm{~g} \mathrm{Jod}=15.55 \% \mathrm{Cl}$.

Ein andermal wurde das Doppelsalz in einem kleinen Kölbchen mit verdünnter $\mathrm{H}_{2} \mathrm{SO}_{4}$ und einem Stückchen Magnesit gekocht und das Destillat in einer Jodkaliumlösung aufgefangen.

$0.2755 \mathrm{~g}$ Substanz entsprechen $0.1527 \mathrm{~g}$ Jod $=15.49 \% \mathrm{Cl}$.

Das Mittel beider Bestimmungen $=15.52 \%$.

Die Bestimmung des Ammoniums geschah als $\mathrm{NH}_{3}$. Dieses wurde mittelst ̈̈tznatronlösung ausgetrieben. Das Doppelsalz wurde mit Schwefelwasserstoffwasser zerlegt, das Bleisulfid abfiltrirt, aus dem Filtrat der überschüssige Schwefelwasserstoff durch Luft verdrängt und dann mit Natronlauge destillirt.

$0.6324 \mathrm{~g}$ Substanz gaben $0.046506 \mathrm{~g} \mathrm{NH}_{3}=7.35 \%=\left(-\mathrm{NH}_{4}\right)=7.79 \%$.

Eine zweite Bestimmung, bei welcher das Ausfällen mit Schwefelwasserstoff unterblieb und das Salz direct mit Natriumhydrat destillirt wurde, gab nahezu dieselben Zahlen.

Ich fand einmal $7 \cdot 78 \%\left(\mathrm{NH}_{4}-\right)$, ein andermal $7 \cdot 68 \%$.

Das Mittel meiner Analysenzahlen stimmt sehr gut mit den Werthen überein, die sich für das Bleitetrachloridchlorammonium $\mathrm{PbCl}_{4} \cdot 2 \mathrm{NH}_{4} \mathrm{Cl}$ berechnen.

\begin{tabular}{|c|c|c|}
\hline & Gefunden & Berechnet \\
\hline$\ldots \ldots \ldots \ldots \ldots$ & $\widehat{45 \cdot 38 \%}$ & $45 \cdot 39 \%$ \\
\hline otal-C1............. & $46 \cdot 49$ & $46 \cdot 67$ \\
\hline Sogenanntes actives $\mathrm{Cl}$. & $15 \cdot 52$ & $15 \cdot 55$ \\
\hline$\left(-\mathrm{NH}_{4}\right) \ldots \ldots \ldots \ldots \ldots$ & $7 \cdot 79$ & $7 \cdot 93$ \\
\hline
\end{tabular}


Classen und $Z$ ahorski finden für diese Doppelverbindung die Formel $2 \mathrm{PbCl}_{4} .5 \mathrm{NH}_{4} \mathrm{Cl}$, d. i. $\mathrm{PbCl}_{4} \cdot 2 \frac{1}{2} \mathrm{NH}_{4} \mathrm{Cl}$.

Ich glaube, dass ihr Salz mit Chlorammonium verunreinigt war. Ich halte es, wie schon erwähnt, für gefährlich, zur Ausfällung concentrirte Chlorammoniumlösung $\mathrm{zu}$ verwenden, selbst wenn man die Vorsicht von Classen und Zahorski gebraucht, einen Überschuss von $\mathrm{PbCl}_{4} \mathrm{zu}$ belassen, denn da die Bildung des Doppelsalzes nicht augenblicklich erfolgt, kann leicht anfänglich ein Theil des $\mathrm{NH}_{4} \mathrm{Cl}$ in Substanz ausfallen, der sich trotz des Überschusses an Bleitetrachlorid mit diesem nicht mehr verbindet.

Ich habe mein Salz lufttrocken zur Analyse verwendet. Das Trocknen bei $+80^{\circ} \mathrm{C}$., wie Classen und Zahorski es vornehmen, ist nicht nöthig, wenn man mit Alkohol gewaschen hat und wohl nicht unbedenklich, da die vollständige Zersetzung der Verbindung schon bei $+120 \mathrm{C}$. eintritt.

Nach meinen Analysen ist das Bleiammoniumtetrachlorid dem Pinksalz analog. Dafür sprechen auch die krystallographischen Verhältnisse, denn nach den oben mitgetheilten Beobachtungen des Herrn Dr. Koechlin krystallisirt die Bleidoppelverbindung ebenso tesseral wie das Pinksalz. Das gleiche Verhalten beider Salze gegen concentrirte Schwefelsäure soll später erwähnt werden.

Das von Classen und $Z$ ahorski beschriebene Verhalten gegen Wasser und andere Agentien kann ich vollständig bestätigen. Es sei zugefügt, dass das Salz in concentrirter Salzsäure ziemlich leicht zu einer klaren Flüssigkeit löslich ist, aus der sich in der Wärme Chlor ausscheidet, ohne dass ein Niederschlag von $\mathrm{PbCl}_{2}$ entstände. Wendet man verdünnte Salzsäure an, so schlägt sich $\mathrm{PbCl}_{2}$ nieder; die Reaction bleibt gleich. Auch concentrirte Salpetersäure löst das Salz in der Kälte ohne Zersetzung. Beim Erwärmen findet Freiwerden von Chlor und Oxyden des Stickstoffes statt, zugleich fällt ein Niederschlag zu Boden, der in Wasser ziemlich leicht löslich ist. Verdünnte $\mathrm{NO}_{3} \mathrm{H}$ wirkt ähnlich, ebenso verdünnte Schwefelsäure.

Ganz anders und entgegen allen bisherigen Erfahrungen setzt sich das Salz mit concentrirter Schwefelsäure um. Hiebei bildet sich das 


\section{Bleitetrachlorid.}

Zur Darstellung desselben trägt man das Doppelsalz in kleinen Portionen in concentrirte reine, mit Eis gekühlte Schwefelsäure ein (etwa in die dreissigfache Menge). Sobald das Doppelsalz mit der Säure in Berührung kommt, entwickelt sich Chlorwasserstoff und es scheidet sich Ammoniumsulfat aus. Bald bemerkt man kleine gelbe Tröpfchen, die sich zuerst an der Oberfläche ansammeln, an Volum zunehmen, dann zu Boden fallen und sich zu einer gelben ölartigen Flüssigkeit vereinigen. Hierbei nimmt man einen eigenthümlichen, an unterchlorige Säure erinnernden Geruch wahr. Mit KJ und Stärkekleister behandeltes Papier färbt sich blau, doch dürfte diese Jodabscheidung einer secundären Reaction, d. i. dem von der Zersetzung der gebildeten neuen Verbindung herrührenden $\mathrm{Cl}$ zuzuschreiben sein. Das Öl wird von der milchigen Suspension von Ammoniumsulfat in Schwefelsäure getrennt und wiederholt mit $\mathrm{H}_{2} \mathrm{SO}_{4}$ geschüttelt, bis diese nach dem Absitzen vollständig klar bleibt und keine feste Substanz wahrzunehmen ist.

In gereinigtem Zustande ist der neue Körper eine vollkommen klare, gelbe, das Licht stark brechende, schwere, doch bewegliche Flüssigkeit, nicht unähnlich dem Antimonpentachiorid, welche analog dem Zinntetrachlorid an feuchter Luft raucht, wobei ein eigenthümlicher, an unterchlorige Säure erinnernder Geruch wahrzunehmen ist, der im Munde einen süsslichen Geschmack hervorruft. Die Verbindung zersetzt sich leicht unter Abscheidung von Bleidichlorid und Freiwerden von Chlor. Unter concentrirter $\mathrm{H}_{2} \mathrm{SO}_{4}$ lässt sich der Körper meist längere $Z$ eit aufbewahren, doch kann es vorkommen, dass unter sonst gleichen. Umständen ziemlich rasch fortschreitende Zersetzung eintritt. Beim Erwärmen (circa bei $+105^{\circ} \mathrm{C}$.) tritt diese explosionsartig auf einmal ein, wobei sich $\mathrm{PbCl}_{2}$ zum Theile in Form von schweren weissen Dämpfen abscheidet und Chlor frei wird.

Die Analyse des Körpers stimmt, wie schon erwähnt wurde, auf das Bleitetrachlorid. 
Das Blei wurde einmal mit wässeriger schwefeliger Säure als Bleisulfat, das anderemal mit Schwefelwasserstoffwasser als Bleisulfid abgeschieden und gewogen.

Behufs Chlorbestimmung wurde einmal wieder durch $\mathrm{SO}_{2}$ zersetzt und im Filtrat der Chlorwasserstoff durch Titration mittelst Silbernitrat bestimmt; das anderemal im Filtrat des Schwefelbleies - nach Verdrängung des $\mathrm{SH}_{2}$ durch einen Luftstrom - mittelst $\mathrm{AgNO}_{3}$ ausgefällt und aus der gefundenen $\mathrm{AgCl}$ - Menge Chlor berechnet.

Bei der Zersetzung mit Schwefelwasserstoffwasser zeigt sich im ersten Augenblick ein röthlicher Körper, wahrscheinlich ein Sulfid mit einem Cl-Reste; dieses wird jedoch durch weiteres Einleiten von $\mathrm{H}_{2} \mathrm{~S}$ vollständig in schwarzes $\mathrm{PbS}$ umgewandelt.

$0.7546 \mathrm{~g} \mathrm{~Pb} \mathrm{Cl}_{4}$ gaben $0.6496 \mathrm{~g} \mathrm{~Pb} \mathrm{SO}_{4}$.

$0.8306 \mathrm{~g} \mathrm{~Pb} \mathrm{Cl}_{4}$ gaben $0.33747 \mathrm{~g} \mathrm{Cl}$.

Es wurden verbraucht $96.05 \mathrm{~cm}^{3} \mathrm{Ag} \mathrm{NO}_{3}$-Lösung (vom Titer $0.0035135 \mathrm{Cl}$ ) $0.5056 g \mathrm{~Pb} \mathrm{Cl}_{4}$ gaben $0.34529 g \mathrm{PbS}$. $0.5056 \mathrm{~g} \mathrm{~Pb} \mathrm{Cl}_{4}$ gaben $0.8223 \mathrm{~g} \cdot \mathrm{Ag} \mathrm{Cl}$.

$\begin{array}{ccccc}1 . & 2 . & 3 . & 4 . & \underbrace{\text { Berechnet für }} \mathrm{Pb} \mathrm{C1}_{+} \\ \mathrm{Pb} \ldots \ldots . \ldots 8 \cdot 79 \% & - & 59 \cdot 13 \% & - & 59 \cdot 33 \% \\ \mathrm{Cl} . \ldots . & 40 \cdot 63 \% & - & 40 \cdot 22 \% & 40 \cdot 67\end{array}$

Die. bei den Analysen gefundenen Werthe stimmen also gut für eine Verbindung der Formel $\mathrm{PbCl}_{4}$ - für das Bleitetrachlorid.

Bleitetrachlorid zersetzt sich beim Erwärmen, wie schon erwähnt wurde, glatt in Chlor und Bleidichlorid. Ich habe diese Reaction auch quantitativ verfolgt.

Ich: wog über $1 \mathrm{~g}$ des Körpers in einem dickwandigen Einschmelzrohr ab, stellte dieses in eine Kältemischung und schmolz es zu. Das Rohr wurde dann mit einem Drahtnetz umhüllt und die Verbindung hierauf durch Wärme zersetzt. Nach dem Abkühlen wurde das an den Wänden der Röhre anhaftende $\mathrm{PbCl}_{2}$ in das eine Ende gebracht. Die grüne Farbe des in derselben befindlichen $\mathrm{Cl}$ trat nun deutlich hervor. Durch Einstellen in eine Kältemischung wurde hierauf die Expansion des Gases geringer gemacht und die Röhre geöffnet, das Chlor 
durch Erwärmen vertrieben, das pulverförmige Bleidichlorid zu Hornblei eingeschmolzen und nach dem Abkühlen gewogen.

Der durch die Abspaltung der zwei $\mathrm{Cl}$-Atome entstandene Gewichtsverlust beträgt $20 \cdot 616 \%$, der theoretische für zwei $\mathrm{Cl}$ berechnete $20 \cdot 305 \%{ }^{.}$

Wie bei dem Bleitetrachloridchlorammonium, so lassen sich auch bei dem $\mathrm{PbCl}_{4}$ jodometrisch nur zwei Chloratome nachweisen, indem bei Einwirkung von $\mathrm{KJ}$ nur zwei J-Atome frei werden. Die Reaction geht. vielleicht in zwei Phasen vor sich:

$$
\begin{aligned}
& \text { I. } \mathrm{Pb} \mathrm{Cl}_{4}+4 \mathrm{KJ}=\mathrm{PbJ}_{4}+4 \mathrm{KCl} \text {. } \\
& \text { II. } \mathrm{PbJ}_{4}=\mathrm{PbJ}_{2}+\mathrm{J}_{2} .
\end{aligned}
$$

Die titrimetische Bestimmung dieser zwei J-Atome wurde wie bei dem Doppelsalze vorgenommen.

$0.6159 g$ der Verbindung wurden in Jodkaliumlösung eingetragen, dann wurde auf einen Liter verdünnt und hierauf in je $250 \mathrm{~cm}^{3}$ die freie Jodmenge mittelst Natriumthiosulfat bestimmt.

Es wurden verbraucht: $76 \mathrm{~cm}^{3} \mathrm{Na}_{2} \mathrm{~S}_{2} \mathrm{O}_{3}$-Lösung (vom Titer $0.00587 \mathrm{~J}$ ). $0.6159 \mathrm{~g} \mathrm{PbCl}_{4}$ gaben $0.44612 \mathrm{~g} \mathrm{~J}=0.124698 \mathrm{~g} \mathrm{Cl}=20.25 \%$, berechnet $20 \cdot 33 \%$.

Titrirt man die das $\mathrm{PbJ}_{2}$ enthaltende Flüssigkeit direct, so erhält man keine verlässlichen Resultate. Der Endpunkt der Reaction ist schwer zu erkennen. Einmal schon desshalb, weil das gelbe $\mathrm{PbJ}_{2}$ den Farbenumschlag der Stärke abschwächt, weiter weil Natriumthiosulfat auf Bleijodid einwirkt. Man erkennt dies deutlich daran, dass das $\mathrm{PbJ}_{2}$ durch überschüssiges $\mathrm{Na}_{2} \mathrm{~S}_{2} \mathrm{O}_{3}$ in ein grünliches Pulver umgewandelt wird und die durch Thiosulfat entfärbte Flüssigkeit nach einiger Zeit wieder blau wird. Bei schnellem Arbeiten erhält man dennoch annähernd gute Zahlen, sonst sind sie immer höher.

Alle quantitativen Versuche sind durch die grosse Unbeständigkeit des Körpers, der sich nur unter concentrirter $\mathrm{H}_{2} \mathrm{SO}_{4}$ unzersetzt aufbewahren lässt, sehr erschwert. Nimmt man ihn

1 Die Gewichtszahlen konnte ich leider in meinen Notizen nicht mehr auffinden, ich fand nur den berechneten procentischen Verlust. 
aus derselben heraus, so findet schon nach einigen Augenblicken Zersetzung statt, wobei eine Trübung der Flüssigkeit wahrzunehmen ist.

Das Entnehmen und Abwägen von Proben ist daher mit Schwierigkeiten verbunden; am leichtesten lässt es sich auf folgende Weise ausführen:

Eine capillare Pipette wird unten rechtwinkelig abgebogen und zugeschmolzen, "durch die Schwefelsäure in das Bleitetrachlorid geführt, dann mit einem Glasstab unten abgebrochen und nun wird vorsichtig aufgesaugt. Die Pipette wird dann herausgenommen, gut abgewischt und ihr Inhalt unter Verzicht auf die ersten und letzten Tropfen in ein Wägeglas gebracht. Auf diese Weise kann man ziemlich rasch wägen und ist sicher, keine Schwefelsäure mitzunehmen.

Das specifische. Gewicht des Bleitetrachlorids ist $3 \cdot 183$ bei $0^{\circ} \mathrm{C}$. Der gefundene Werth ist das Mittel der Zahlen von $z$ Wei Bestimmungen, die alle bei $0^{\circ} \mathrm{C}$. ausgeführt wurden.

I. Piknometer leer ........23:868 g

$$
\begin{aligned}
& +-\mathrm{H}_{2} \mathrm{O}\left(0^{\circ}\right) \ldots \ldots 31.575 \mathrm{~g} \\
& +\mathrm{PbCl}_{4}\left(0^{\circ}\right) \ldots .48 \cdot 390 \mathrm{~g}
\end{aligned} \quad \text { sp. G. }=3 \cdot 182
$$

II. Piknometer leer $\ldots \ldots \ldots \ldots 22 \cdot 452 \xi$

$$
\begin{aligned}
& +\mathrm{H}_{2} \mathrm{O}\left(0^{\circ}\right) \ldots \ldots 26 \cdot 9025 \mathrm{~g} \quad \text { sp. G. }=3 \cdot 184 \\
& +\mathrm{PbCl}_{4}\left(0^{\circ}\right) \ldots \ldots 36 \cdot 6225 \mathrm{~g}
\end{aligned}
$$

Durch Kältemischungen kann man die Verbindung zum Gefrieren bringen. Sie bildet dann eine gelbliche, durchscheinende, krystallinische Masse, welche bei ungefähr - $15^{\circ} \mathrm{C}$. schmilzt. Erwärmt, zersetzt sich der Körper in der schon. angegebenen Weise, șobald die Temperatur eine gewișse Höhe erreicht hat. Unter Schwefelsäure hält er sich jedoch beim Erwärmen ziemlich indifferent. Erhitzt man ihn mit $\mathrm{H}_{2} \mathrm{SO}_{4}$ in einer Retorte, welche ein trockener Chlorstrom passirt, so geräth er in eine dem Sieden ähnliche Bewegung, und in eine gekühlte Vorlage gehen sogar einige Tropfen der unzersetzten Verbindung über. Erreicht die Temperatur aber ungefähr $105^{\circ} \mathrm{C}$, so findet eine explosionsartige Zersetzung statt. Es entweicht Salzsäure und Bleichlorid, beziehungsweise Bleisulfat scheiden sich in der Schwefelsäure und als Rauch im Hals der Retorte ab. 
Analog dem $\mathrm{SnCl}_{4}$ scheint auch das Bleitetrachlorid mit Wasser Hydrate $z u$ bilden. Bringt man den Körper auf einem Uhrgläschen, das auf Eis liegt, mit einem Tropfen kalten Wassers zusammen, so bildet sich ein krystallinischer gelber Körper. Nimmt man das Uhrglas vom Eise weg, so findet bald Zersetzung statt, die von einem knackenden Geräusch begleitet ist. Dabei werden Theilchen der Substanz aus dem Uhrglas herausgeschleudert. Fügt man mehr gekühlten Wassers hinzu, so löst sich der Körper klar auf. Beim Erwärmen findet Zersetzung unter $\mathrm{Abscheidung}$ von $\mathrm{PbCl}_{2}$ und Freiwerden von Chlor statt.

Viel $\mathrm{H}_{2} \mathrm{O}$ zerlegt das $\mathrm{PbCl}_{4}$, sowie das Doppelsalz $\mathrm{PbCl}_{4} .2 \mathrm{NH}_{4} \mathrm{Cl} z \mathrm{zu}$ Bleihyperoxyd und Salzsäure.

Ätznatronlösung scheidet sofort $\mathrm{PbO}_{2}$ ab. Von Säuren lösen viele das $\mathrm{PbCl}_{4} \mathrm{zu}$ gelben Flüssigkeiten auf. Diese zersetzen sich durch Wärme, wobei Chlor frei wird.

Concentrirte Salzsäure löst die Verbindung in jedem Verhältniss. Ammoniumchlorid fällt dann das früher beschriebene Bleitetrachloridchlorammonium, und dieses gibt mit concentrirter $\mathrm{H}_{2} \mathrm{SO}_{4}$ wieder flüssiges Bleitetrachlorid.

Mit kleinen Mengen gekühlter Salzsäure entsteht ein krystallinischer Körper, vielleicht ein Hydrochlorid von der Formel $\mathrm{PbCl}_{6} \mathrm{H}_{2}$.

Concentrirte Schwefelsäure wirkt auf Bleitetrachlorid nicht ein.

Interessant ist die Einwirkung von metallischem Natrium und Kalium. Beide Metalle schwimmen auf dem Bleitetrachlorid, ohne dass eine Reaction zu bemerken wäre, sie bedecken sich lediglich mit einer braunen Substanz, die $\mathrm{PbO}_{2}$ sein dürfte. Erwärmt man $\mathrm{Na}$ mit $\mathrm{PbCl}_{4}$ in einer Eprouvette, so zersetzt sich zuerst das Tetrachlorid in der bekannten Weise, dann tritt das $\mathrm{Na}$ in Action. Beim $\mathrm{K}$ tritt beim Erwärmen Explosion ein.

Quecksilber scheint auf Bleitetrachlorid in der Kälte nicht einzuwirken.

Die Isolirung des Bleitetrachlorids hat nicht nur das Interesse, dass sie die Tetravalenz des Bleies in anorganischen 
Verbindungen ausser $Z$ weifel setzt, sondern sie ist auch für die Beziehungen zwischen Blei und Zinn werthvoll.

Beide Metalle geben zwei Chloride der Formel $\mathrm{RCl}_{2}$ und $\mathrm{RCl}_{4}$.

Die Dichloride sind krystallinische, sehr beständige Körper, lassen sich beide zu dunkelgelben Flüssigkeiten einschmelzen, welche beim Abkühlen zu festen, glänzenden, schweren Krystallmassen erstarren. Beide sind in der Hitze flüchtig.

Die höheren Chloride sind beide flüssige Körper, rauchen an der Luft, sind beide flüchtig, doch zeigt sich beim $\mathrm{PbCl}_{4}$ die Tendenz, zwei Cl-Atome abzuspalten und die niedere Verbindung $\mathrm{zu}$ bilden, während das Zinntetrachlorid verhältnissmässig sehr beständig ist und sich leicht destilliren lässt, was bei $\mathrm{PbCl}_{4}$ nur in geringem Masse der Fall ist. Beide Chloride geben mit Ammoniumchlorid Doppelsalze, die isomorph sind.

Interessant ist das Verhalten der Chloride gegen Wasser.

Die beiden Zinnchloride verbinden sich mit grosser Begierde mit Wasser. Zinndichlorid dient bekanntlich als wasserentziehendes Mittel. Das Tetrachlorid raucht an feuchter Luft und bildet zahireiche Hydrate.

Bleidichlorid ist nicht hygroskopisch; Bleitetrachlorid zeigt dasselbe Verhalten wie das $\mathrm{SnCl}_{4}$, doch in schwächerem Masse. $\mathrm{PbCl}_{4}$ raucht auch an der Luft, doch schwächer und dürfte es auch Hydrate bilden.

Zinn und Blei stehen einander demnach näher, als bis jetzt angenommen wurde. Das zeigt auch viel deutlicher ein Verhalten, auf das ich später zurückkommen werde.

Die Abscheidung des Bleitetrachlorids aus dem Bleitetrachloridchlorammonium durch concentrirte Schwefelsäure, sowie der Umstand, dass man das $\mathrm{PbCl}_{4}$ unter $\mathrm{H}_{2} \mathrm{SO}_{4}$ aufbewahren kann, ohne dass eine Einwirkung zu bemerken wäre, scheint mir eine den bisherigen Erfahrungen entgegenlaufende Thatsache.

In allen Fällen, wo concentrirte Schwefelsäure auf Metallchloride in Substanz einwirkt, wird Chlorwasserstoff unter Bildung von Sulfaten ausgetrieben. Das $\mathrm{PbCl}_{4}$ kann mit einem Überschusse concentrirter $\mathrm{H}_{2} \mathrm{SO}_{4}$ gewonnen und selbst erwärmt werden, ohne dass eine Reaction wahrzunehmen wäre. 
Um festzustellen, ob dieses ungewöhnliche Verhalten nur bei dem Bleitetrachlorid oder auch bei anderen Chloriden angetroffen werde, habe ich die Einwirkung der concentrirten Schwefelsäure auch auf andere Metallchloride untersucht und beabsichtige, diese Versuche noch fortzusetzen. Bis jetzt ist es mir zwar nicht gelungen, eine Gesetzmässigkeit im Verhalten der Metallchloride gegen Schwefelsäure zu finden, doch konnte ich feststellen, dass bestimmt $\mathrm{Sn} \mathrm{Cl}_{4}$ und wahrscheinlich auch $\mathrm{GeCl}_{4}$ ebenso indifferent gegen concentrirte $\mathrm{H}_{2} \mathrm{SO}_{2}$ sind, wie $\mathrm{PbCl}_{4}$.

Wie auf Bleichlorid, so wirkt Schwefelsäure auch auf Zinndichlorid. Wasserfreies, frisch dargestelites $\mathrm{SnCl}_{2}$ zersetzt sich bei Einwirkung von $\mathrm{H}_{2} \mathrm{SO}_{4}$ schon in der Kälte. Beim Erhitzen wird die HCl-Entwicklung stürmisch. und bald tritt vollständige Lösung ein.

Auf $\mathrm{SnCl}_{4}$ wirkt Schwefelsäure nicht ein. Auch beim Erhitzen ist die Einwirkung eine ziemlich schwache. Das $\mathrm{SnCl}_{4}$ kann mit $\mathrm{H}_{2} \mathrm{SO}_{4}$ überschichtet, bei vorsichtiger Manipulation destillirt werden.

Trägt man das dem $\mathrm{PbCl}_{4} \cdot 2 \mathrm{NH}_{4} \mathrm{Cl}$ analoge Pinksalz $\mathrm{SnCl}_{4} .2 \mathrm{NH}_{4} \mathrm{Cl}$ in concentrirte Schwefelsäure ein, so scheidet sich $\mathrm{Sn} \mathrm{Cl}_{4}$ ab. Es entwickelt sich ebenfalls. Chlorwasserstoff in grossen Mengen, die Abscheidung dauert jedoch etwas länger als beim $\mathrm{PbCl}_{4}$. Nach Verlauf von circa $10-12$ Stunden findet man am Boden des Gefässes farblose Tröpfchen einer Flüssigkeit, die $\mathrm{SnCl}_{4}$ sind und beim Erwärmen in eine gekühlte Vorlage überdestillirt werden können. Die Zersetzung des Pinksalzes geht wie die der analogen Bleiverbindung vor șich.

$$
\mathrm{SnCl}_{4} \cdot 2 \mathrm{NH}_{4} \mathrm{Cl}+\mathrm{H}_{2} \mathrm{SO}_{4}=\mathrm{SnCl}_{4}+\left(\mathrm{NH}_{4}\right)_{2} \mathrm{SO}_{4}+2 \mathrm{HCl} \text {. }
$$

Durch die Munificenz des Herrn geh. Hofraths Prof. Dr. Clemens Winkler, welcher auf Ansuchen des Herrn Prof: Dr. Skraup mir nahezu $2 g$ Germaniumtetrachlorid zur Verfügung stellte, wurde ich in die angenehme Lage versetzt, auch einige Versuche mit dieser Verbindung vorzunehmen.

Es ist mir ein besonderes Vergnügen, Herrn Prof. Dr. Cl. Winkler für seine gütige Spende bestens zu danken.

Concentrirte Schwefelsäure wirkt auf Germaniumtetrachlorid in der Kälte nicht ein. Auch beim Erhitzen ist keine 
besondere Einwirkung wahrzunehmen. In eine mit Schwefelsäure beschickte Retorte gebracht, schwamm das $\mathrm{GeCl}_{4}$ auf der Säure und destillirte beim Erwärmen unverändert über. Es war keine Salzsäureentwicklung wahrzunehmen und in der Schwefelsäure konnte nach dem Abrauchen etc. kein Ge nachgewiesen werden. Nach diesem Verhalten war zu schliessen, dass sich das $\mathrm{GeCl}_{4}$ aus einem dem Pinksalz analogen Doppelsalze mit Schwefelsäure werde ausscheiden lassen. Ich habe mich vergebens bemüht, dieses bisher nicht bekannte Salz darzustellen.

Eisenchlorür und Eisenchlorid, mit aller Sorgfalt bei Abschluss jeder Feuchtigkeit bereitet und mit concentrirter Schwefelsäure übergossen, reagiren schon in der Kälte unter Ausscheidung von Salzsäuredämpfen anscheinend gleich energisch.

Antimon-Trichlorid und Pentachlorid, durch Destillation (das Pentachlorid nach der Sättigung mit Chlor im Vacuum) gereinigt, werden, wenn sie mit concentrirter Schwefelsäure übergossen, im Vacuum destillirt werden, leicht angegriffen. In der Kälte ist die Einwirkung auf das Trichlorid schwach, Pentachlorid wird leichter angegriffen. Im Allgemeinen ist das Trichlorid die beständigere Verbindung. Die Reactionen scheinen hier übrigens, besonders beim Pentachlorid, complicirter zu verlaufen.

Es sei noch des Versuches erwähnt, das Bleitetrabromid oder ein Doppelsalz desselben mit $\mathrm{NH}_{4} \mathrm{Br}, \mathrm{KBr}$ oder $\mathrm{NaBr}$ darzustellen.

Es wurde reines Bleibromid mit rauchender Bromwasserstoffsäure und mit Brom behandelt, ohne dass die Bildung eines neuen Körpers wahrgenommen werden konnte.

Bei einem weiteren Versuche wurde reines, frisch bereitetes Bleihyperoxyd mit rauchender Bromwasserstoffsäure unter Kühlung behandelt. Auch dieser Versuch blieb resultatlos.

Endlich habe ich auf Bleibromid genügende Mengen Brom bei Gegenwart eines Überschusses an wässeriger Bromkaliumlösung einwirken lassen.

Es lässt sich Anfangs und bei gewöhnlicher Temperatur keine Einwirkung beobachten, stellt man jedoch das Gemisch 
in Eis ein, so bemerkt man nach 10-12 Stunden die Bildung eines rothbraunen krystallinischen Körpers. Dieser zersetzt sich, sobald er aus der Flüssigkeit herausgenommen wird, rasch unter Freiwerden von Brom. Im weissen Rückstande kann man Bleibromid und Kalium nachweisen, woraus auf ein Bleitetrabromidbromkalium zu schliessen wäre.

Zweifellos lassen sich organische Verbindungen von Bleitetrabromid und Bleitetrajodid, wie aus den Versuchen von Classen und Zahorski hervorgeht, viel leichter darstellen.

Zum Schlusse fühle ich mich veranlasst, Herrn Prof. Dr. Zd. H. Skraup für die vielfache freundliche Unterstützung, die er mir bei der.Ausführung dieser Arbeit zu Theil werden liess, auch an dieser Stelle meinen verbindlichsten Dank auszusprechen. 